\title{
Awareness during Cardiopulmonary Resuscitation
}

\author{
Ali Asghar ${ }^{1}$, Bushra Salim², Saqiba Tahir ${ }^{3}$, Fahad Islam ${ }^{4}$, Muhammad F Khan ${ }^{5}$
}

\begin{abstract}
Checking responsiveness is the mainstay in cardiopulmonary resuscitation (CPR). It is rare in the clinical situation when the patient requires resuscitation despite the presence of wakefulness. We report a case in which the patient presented with flat arterial line and absence carotid pulse while he was awake. A thorough literature review will also be discussed.

Keywords: Cardiopulmonary resuscitation, Life support, Responsiveness.

Indian Journal of Critical Care Medicine (2020): 10.5005/jp-journals-10071-23345
\end{abstract}

\section{INTRODUCTION}

Cardiac arrest (CA) is almost always associated with loss of consciousness. Consciousness during $\mathrm{CA}$ and cardiopulmonary resuscitation (CPR) is rare, ${ }^{1-3}$ but it is most likely with high-quality CPR with few interruptions, adequate depth of chest compression, and use of mechanical devices for chest compression. We describe the course of a patient who was awake and communicating during CPR. However, despite timely diagnosis and early initiation of CPR, the patient died.

There are no recommendations on the management of consciousness or awareness during CPR from the International Liaison Committee on Resuscitation (ILCOR). ${ }^{4}$ All guidelines for the resuscitation of $C A$ recommend checking responsiveness before starting CPR if the patient is unresponsive. Both basic life support and advanced cardiac life support recommend starting cardiac compressions if the patient shows no responsiveness with absent pulse during carotid check.

\section{Case Description}

A 62-year-old man with diabetes, hypertension, and ischemic heart disease presented with abdominal pain, abdominal distention, absolute constipation, and weakness, which began 2 days prior to his presentation. He also reported $10 \mathrm{~kg}$ weight loss in the preceding 2 months.

Intestinal obstruction secondary to mass in cecum was diagnosed on computed tomography of the abdomen. So, emergency exploratory laparotomy, total colectomy, and ileostomy were performed. The patient was transferred to the intensive care unit (ICU) postoperatively and extubated onto bilevel positive airway pressure (BIPAP) 24 hours later. He remained hemodynamically stable but BIPAP dependent for the next 24 hours. While the ICU consultant was on morning round his nurse noticed flattening of the right radial arterial line trace. However, the patient was awake and speaking to his son, describing mild chest tightness. Sinus rhythm was observed on electrocardiogram monitoring. The arterial line and transducer were flushed and assessed for faults while the left brachial and carotid pulses were palpated.

Pulses were not palpable, and CPR was started to manage pulseless electrical activity. During CPR, the patient's eyes remained open and he obeyed simple commands (e.g., to open his mouth) despite the compression-based arterial wave form which became flat during pulse check. An endotracheal tube was inserted without
${ }^{1-5}$ Department of Anesthesiology, Aga Khan University Hospital, Karachi, Pakistan

Corresponding Author: Muhammad F Khan, Department of Anesthesiology, Aga Khan University Hospital, Karachi, Pakistan, Phone: +92 34864623, e-mail: mfaisal.khan@aku.edu

How to cite this article: Asghar A, Salim B, Tahir S, Islam F, Khan MF. Awareness during Cardiopulmonary Resuscitation. Indian J Crit Care Med 2020;24(2):136-137.

Source of support: Nil

Conflict of interest: None

sedation or analgesia. The patient lost consciousness after the third cycle of CPR. Return of spontaneous circulation was not achieved despite 40 minutes of CPR. So, the patient's family was informed, further attempts at resuscitation were stopped and death was declared.

During CPR, several healthcare providers were involved but none of them had ever experienced such situation in which the patient was fully awake during CPR. It was distressing to all of them, and they were uncertain about the management of patient during CPR related to his consciousness. Either such patients should be sedated/restrained or not.

\section{Discussion}

Patient awareness during CA and CPR is thought to be extremely rare. It can cause diagnostic uncertainty and delay management. Moreover, it may be extremely distressing for the patient, their family, and healthcare professionals.

The delivery of high-quality CPR with minimum interruptions, adequate depth of chest compression, and the use of mechanical devices can generate $20-30 \%$ of the prearrest cardiac output. ${ }^{5}$ This can maintain cerebral perfusion pressure and allow consciousness. ${ }^{6}$

Manual chest compression rarely produces a mean arterial pressure (MAP) of over $40 \mathrm{~mm} \mathrm{Hg} .{ }^{4}$ Two cases have reported that patients were awake with MAP over $50 \mathrm{~mm} \mathrm{Hg} .{ }^{4}$ Conversely, there are reports of higher MAP being achieved during CPR but patients remained unconsciousness. ${ }^{7}$ Individual factors, such as autoregulation of cerebral blood flow, ${ }^{8}$ ischemic threshold, ${ }^{3}$ and comorbidities, ${ }^{2}$ influence brain oxygenation and may contribute to CPR-induced consciousness. Furthermore, early CPR and skilled CPR 
by trained personnel (e.g., in-hospital witnessed arrest) are likely to be key factors leading to CPR-induced consciousness. ${ }^{9}$

Lundsgaard et al. reviewed seven papers on consciousness during CPR and concluded that awareness during CPR or CPRinduced consciousness remains rare but is increasingly reported. Two decades ago Martens and Mullie asked whether a guideline was required for sedation during CPR. ${ }^{5}$ Yet, there are still no recommendations on the management of consciousness or awareness during CPR from the ILCOR. ${ }^{4}$

Further research is needed before definite guidance can be given. Local advice should be followed regarding the use of sedation and analgesia during CPR. ${ }^{10}$

In the book Life after life, Moody ${ }^{11}$ described the experiences of 150 people who had been close to death. ${ }^{4}$ He coined the term near-death experiences for the recurring features reported by these survivors. Moody's observations were not only limited to the survivors of CA but also included people considered sufficiently ill to have died without medical intervention. Near-death experiences have been reported in up to $12-18 \%$ of CA survivors. ${ }^{12}$

Parnia et al. conducted a 4-year prospective multicenter observational study on awareness during CPR. They interviewed 140 CA survivors to measure ${ }^{1}$ awareness/memories during CA and ${ }^{2}$ objectively verified claims of awareness using specific tests. Of their cohort, $46 \%$ had memories with seven major cognitive themes as follows: fear, animals/plants, bright light, violence/persecution, déjà vu, family, and recalling events post CA. Importantly, 2\% described awareness with explicit recall of "seeing" and "hearing" actual events related to their resuscitation. One patient had a verifiable period of conscious awareness at a time when cerebral function was not expected. They concluded that CA survivors commonly experience a broad range of cognitive themes, with $2 \%$ exhibiting full awareness. Other recent studies have also suggested that consciousness may be present despite clinically undetectable consciousness. This may contribute to post-traumatic stress disorder (PTSD) and other cognitive deficits post CA. ${ }^{13} \mathrm{Gray}^{14}$ also reported a case who was aware during CPR. He survived and reported chest discomfort during CPR.

Now question arises whether these patients should be sedated or not during CPR. Because Olaussen et al. ${ }^{15}$ reported two associations with CPR-induced consciousness (CPRIC). It was independently associated return of spontaneous consciousness with and survival to hospital discharge and those patients who received opiates, benzodiazepines, or muscle relaxants during CPR took longer time to obtain return of spontaneous consciousness and were less likely to survive to discharge.

\section{Conclusion}

As more advanced paramedic services adopt evidence-based and clinically proven resuscitation practices, our concern is there will be a growing number of patients who will be aware during resuscitation after $C A$. This necessitates further research, education, and training of hospital care providers in the management of patients experiencing CPR-induced consciousness. We also need to develop guidelines to handle such situations in a better way.

\section{References}

1. Olaussen A, Shepherd M, Nehme Z, Smith K, Bernard S, Mitra B. Return of consciousness during ongoing cardiopulmonary resuscitation: a systematic review. Resuscitation 2015;86(1):44-48. DOI: 10.1016/j. resuscitation.2014.10.017.

2. Jespersen SN, Ostergaard L. The roles of cerebral blood flow, capillary transit time heterogeneity, and oxygen tension in brain oxygenation and metabolism. J Cereb Blood Flow Metab 2012;32(2):264-277. DOI: 10.1038/jcbfm.2011.153.

3. Bandera E, Botteri M, Minelli C, Sutton A, Abrams KR, Latronico N. Cerebral blood flow threshold of ischemic penumbra and infarct core in acute ischemic stroke: a systematic review. Stroke 2006;37(5): 1334-1339. DOI: 10.1161/01.STR.0000217418.29609.22.

4. Nolan JP, Soar J, Zideman DA, et al. European resuscitation council guidelines for resuscitation 2010 section 1. Executive summary. Resuscitation 2010;81(10):1219-1276. DOI: 10.1016/ j.resuscitation.2010.08.021.

5. Martens $P$, Mullie A. Sedation during and after CPR-efforts: is it worth a guide-line? Resuscitation 1995;29(3):223-224. DOI: 10.1016/03009572(94)00836-5. http://hdl.handle.net/10822/884731.

6. Tobin JM, Mihm FG. A hemodynamic profile for consciousness during cardiopul-monary resuscitation. Anesth Analg 2009;109(5): 1598-1599. DOI: 10.1213/ANE.0b013e3181b89432.

7. McDonald JL. Systolic and mean arterial pressures during manual and mechan-ical CPR in humans. Ann Emerg Med 1982;11(6):292-295. DOI: 10.1016/S0196-0644(82)80125-X.

8. Moppett IK, Hardman JG. Modeling the causes of variation in brain tissue oxygenation. Anesth Analg 2007;105(4):1104-1112. DOI: 10.1213/01.ane.0000281934.99076.89.

9. Bihari S, Rajajee V. Prolonged retention of awareness during cardiopulmonary resuscitation for asystolic cardiac arrest. Neurocrit Care 2008;9(3):382-386. DOI: 10.1007/s12028-008-9099-2.

10. Lundsgaard RS, Lundsgaard KS. BET 2: Pain management in patients who show awareness during CPR. Emerg Med J 2019;36(4):249-250. DOI: 10.1136/emermed-2019-208599.2.

11. Moody RA. Life after life: the investigation of a phenomenon - survival of bodily death, 1st ed., Atlanta: Mockingbird Books; 1975. p. 125.

12. Greyson B. The near-death experience scale. Construction, reliability, and valid-ity. J Nerv Ment Dis 1983;171(6):369-375. DOI: 10.1097/00005053-198306000-00007.

13. Parnia S, Spearpoint K, de Vos G, Fenwick P, Goldberg D, Yang J, et al. AWARE-awareness during resuscitation-a prospective study. Resuscitation 2014;85(12):1799-1805. DOI: 10.1016/ j.resuscitation.2014.09.004.

14. Gray R. Consciousness with cardiopulmonary resuscitation. Can Fam Physician 2018;64(7):514.

15. Olaussen A, Nehme Z, Shepherd M, et al. Consciousness induced during cardiopulmonary resuscitation: an observational study. Resuscitation 2017;113:44-50. DOI: 10.1016/j.resuscitation.2017.01.018. 